\title{
Effects of green tea and bisphosphonate association on dental socket repair of rats
}

\author{
Edson Yoshihiro Mada ${ }^{\mathrm{a}}$, Alana Claro Cunha Santos ${ }^{\mathrm{a}}$, Angelica Cristina Fonseca ${ }^{\mathrm{b}}$, \\ Claudia Cristina Biguetti ${ }^{\mathrm{b}}$, Fernando Tozze Alves Neves ${ }^{\mathrm{a}}$, Patrícia Pinto Saraiva ${ }^{\mathrm{a}}$, \\ Mariza Akemi Matsumoto ${ }^{\mathrm{a}, \mathrm{c}, *}$ \\ a Department of Health Sciences, Sagrado Coração Univesity - USC, Rua Irmã Arminda 10-50, 17011-160, Bauru, SP, Brazil

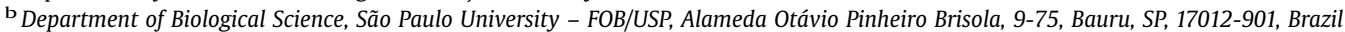 \\ ' Department of Basic Sciences, São Paulo State University (Unesp), School of Dentistry, Araçatuba, Rua José Bonifácio 1193, 16015-050, Araçatuba, SP, Brazil
}

\section{A R T I C L E I N F O}

\section{Article history:}

Received 29 March 2016

Received in revised form 29 November 2016 Accepted 1 December 2016

\section{Keywords:}

Bisphosphonates

Bone repair

Green tea

Immunohistochemistry

Osteonecrosis

Rats

\begin{abstract}
A B S T R A C T
Objectives: To evaluate the effects of green tea intake and zoledronic acid intravenous therapy on teeth socket repair.

Design: Sixty male albinus Wistar rats were divided into 4 groups: C-Control, intravenous (IV) $0.9 \%$ saline solution (SS), GT-1\% green tea in drinking water and IV SS, BP-IV zoledronic acid (BP), and BP + GT-IV BP and $1 \%$ green tea. $0.035 \mathrm{mg} / \mathrm{kg}$ of BP was administered every two weeks. After ten weeks, right upper molars were extracted and the green tea started to be offered for GT and BP + GT. After 7, 14, and 28 days the animals were euthanized.

Results: Histopathology analysis revealed lack of socket repair in BP and BP + GT groups, which presented significant increased number of polimorphonuclear leukocytes at day 28, in comparison with $\mathrm{C}$ $(\mathrm{p}<0.05)$. No significant differences were detected between $\mathrm{C}$ and the experimental groups at the same period $(\mathrm{p}<0.05)$ when considering mononuclear leukocytes. Immunolabeling revealed that the association of BP and GT caused a slight disturbance in OPG/RANKL system and retarded Runx-2 labeling. Although strong TRAP labeling was observed, most of the positive cells in BP and BP + GT groups were not located on bone surface.

Conclusions: Socket healing of rats treated with BP and regular drinking green tea presented no relevant differences in comparison to those treated with BP alone.
\end{abstract}

(C) 2016 Elsevier Ltd. All rights reserved.

\section{Introduction}

Nitrogen-containing bisphosphonates (nBPs) are largely known for their high efficiency in treating osteolytic disorders, from osteoporosis to bone metastasis (Russell, 2011). After their administration, nBPs are almost immediately deposited in bone and their metabolism dependends on the dynamics of bone resorption (Fisher, Rodan, \& Reszka, 2000; Cremers \& Papapoulos, 2011). Low serum concentration and strong bone matrix incorporation of nBPs explain their low toxicity (Lawson et al., 2010). However, some factors influence drug absorption by the organism, such as dose, method of administration, and periodicity (Hadji, 2011), guided by the pathology to be treated.

\footnotetext{
* Corresponding author at: Rua José Bonifácio 1193, 16015-050, Araçatuba, SP, Brazil.

E-mail address: mariza.matsumoto@foa.unesp.br (M.A. Matsumoto).
}

Most potent nBPs, as zoledronic acid and pamidronate, are intravenously administered (IV), usually indicated for metastatic cancer, multiple myeloma, and severe hypercalcemia (Marx, Sawatari, Fortin, \& Broumand, 2005) due to their higher affinity and deposition in bone (Gutta \& Louis, 2007). Evidences have been strongly confirmed that these drugs are internalized by the osteoclasts during bone resorption and inhibit farnesyl pyrophosphate synthase, which consequently prevents farnesyl pyrophosphate (FPP) and geranylgeranylphosphate (GGPP) synthesis (Rogers, Crockett, Coxon \& Mönkkönen, 2011). The lack of these molecules, mainly GGPPs, prevents the prenylation of small GTPases signaling proteins that regulate osteoclast function, such as cytoskeletal organization, membrane ruffling and apoptosis (Coxon \& Rogers, 2003). On the other side, nBPs anti-apoptotic effects on osteocytes and osteoblasts have been observed (Loiselle, Jiang, \& Donahue, 2013). However, this efficient capacity of inhibiting bone resorption and maintenance of osteocytes results in a significant change in whole tissue characteristics, since its 
natural turnover is disrupted. In long term, bone matrix becomes more brittle, osteocytes turn old and vascularization decrease, resulting in a poor reactive tissue favoring some adverse conditions as osteonecrosis of the jaws (Marx, 2003). In addition, considering the mechanism of action of anti-RANKL drugs, a recent in vitro study on nBP zoledronic acid demonstrated similarity between them. It was revealed that zoledronate inhibited genes related to RANKL- induced osteoclast differentiation, as of the transcription factor of the nuclear factor of the activated T cell, NFATC1, and CAII, which mediates the hormones responsible for bone resorption and osteoclast formation (Nakagawa et al., 2015).

It is known that alternative therapies represent an important and increasing role in prevention and treatment of a number of pathologies from diverse etiologies (Tomata et al., 2012). Antioxidant effects of poliphenols compounds (catechins) derived from plants as green tea (Camellia sinensis) have been proved to be effective in various conditions, including bone tissue disorders (Shen, Yeh, Cao, Chyu, \& Wang, 2011). Considering total catechin amount in green tea, epigallocatechin-3-gallate (EGCG) corresponds for up to 59\% (Singh, Akhtar, \& Haqqi, 2010). Studies have demonstrated the direct influence of catechins in different pathways of osteoclastic differentiation and activation, including via metalloproteases (Oka et al., 2012; Yun et al., 2004) and RANKL (Sato \& Takayanagi, 2006), as well as in the improvement of osteoblastic differentiation and activation via Runx-2 (Runtrelated transcription factor-2) (Chen, Ho, Chang, Hung, \& Wang, 2005). In pathologic condition, such as osteoporosis, EGCG effects seem to inhibit inflammation mediated by eicosanoids, as cyclooxygenase 2 (COX-2), lipoxygenase and nitric oxyde synthase (Tipoe, Leung, Hung, \& Fung, 2007). In both situations the beneficial effects of green tea polyphenols in bone tissue is evident.

Numerous worldwide population under nBPs therapy and the imminent risk of osteonecrosis of the jaws have raised the necessity of the development of a number of clinical and experimental studies in order to characterize and elucidate it histopathology. In the field of experimental researches, different animals are used and rats are the majority (Barba-Recreo et al., 2014; Sharma, Hamlet, Pectu, \& Ivanovski, 2013). Despite the systemic commitment that the patients under nBPs therapy usually present, when it comes to animal models this is not a mandatory condition for inducing osteonecrosis of the jaws, as proved by previous reports (Biasotto et al., 2010; Hokugo et al., 2010; Marino et al., 2012; Senel et al., 2010). Therefore, considering the high consumption of both substances by world population and the risk of osteonecrosis, their simultaneous ingestion was investigated in this study in order to observe their effects on socket bone healing after teeth extractions, and serve as an indicative on how simple daylife associations can interfere in healing mechanisms.

\section{Materials \& methods}

\subsection{Study design}

The present study has been approved by the Ethical Committee for Animal Care (protocol 037/2012) of Bauru School of Dentistry, São Paulo University, Bauru, Brazil. All experimental protocols involving animals followed the National Institutes of Health guide for the care and use of Laboratory animals (NIH), as well as Brazilian Society of Laboratory Animal Science (COBEA). Sixty male Wistar rats, mean weight $300 \mathrm{~g}$, average age of 5 months, were kept in controlled environment of $21-22^{\circ} \mathrm{C}$ temperature and $12 \mathrm{~h}$ lightdark ycles, with food and water ad libitum, except for the animals treated with green tea.

\subsection{Total phenol compounds analysis from $1 \%$ green tea infusion}

Green tea infusion was daily prepared and its total phenol compounds was analyzed under spectrophotometry according to Folin-Ciocalteu method (Mah et al., 2014), using galic acid as pattern. Three different temperatures were observed, 40, 60 and $100{ }^{\circ} \mathrm{C}$, under 5 and $10 \mathrm{~min}$-infusion. The combination that resulted in the highest phenolic compounds concentration was chosen for green tea preparation in the present study.

\subsection{Treatment protocols}

The animals were divided into four groups ( $\mathrm{n}=15$ each), according to the treatment, as follows: Group C: negative control, intravenous (IV) $0.9 \%$ saline solution (SS), Group GT: $1 \%$ green tea (Mate Leão, The Coca Cola Company, Curitiba, Brazil) in drinking water and IV 0.9\% SS, Group BP: IV zoledronic acid (BP) (Zometa, Novartis Pharma AG, Basel, Switzerland), Group BP +GT: $1 \%$ green tea, and IV BP.

The animals of groups $\mathrm{BP}$ and $\mathrm{BP}+\mathrm{GT}$ were treated with $0.035 \mathrm{mg} / \mathrm{kg}$ of zoledronic acid IV (Hokugo et al., 2010), every two weeks. Those of groups GT and BP+GT received $20 \mathrm{ml}$ of $1 \%(\mathrm{w} / \mathrm{v})$ green tea in drinking water (de Moraes et al., 2004), daily prepared in which $1 \mathrm{~g}$ of tea leaves were infused in $100 \mathrm{ml}$ at $100^{\circ} \mathrm{C}$ filtered water for $5 \mathrm{~min}$. After five doses of BP, all animals had their right upper molars extracted, from when they started receiving green tea.

\subsection{Surgical procedure}

All animals underwent surgical procedure for dental extraction following strict asseptic protocol. Sedation was induced with intramuscular (IM) administration of 1\% ketamine (Frankotar, Virbac Ltda, São Paulo, Brazil) along with $2 \%$ chloridrate of xylazine (Virbaxyl 2\%, Virbac Ltda, São Paulo, Brazil) in the recommended dose according to each animal weight. Once sedated, antisepsy with topic $1 \%$ polyvinylpyrrolidone was performed before dental extractions. A delicate sindesmotomy was made in the gingival sulcus of the upper right molars with $3 S$ hollemback spatula, followed by teeth luxation with an apical elevator (EW1, Hu-Friedy, Chicago, USA) adapted in medial and distal bone crests, until total teeth luxation was achieved for further extraction with a Backaus clamp that perfectly adapts in molars furcation, in order to avoid root fractures. After that, sockets were cleaned with sterile $0.9 \%$ saline solution and spontaneously filled with blood clot.

\subsection{Histologic procedures}

After 7, 14, and 28 days from tooth extraction, five animals of each period and group were euthanized with anesthetic overdose for specimens' retrieval, which were immediately immersed in 10\% formaline (Merck, Darmstadt, Germany) for $48 \mathrm{~h}$. Macroscopical aspect of the extraction sites was registered (Fig. 1). After fixation, specimens were washed in tap water for $24 \mathrm{~h}$, immersed in buffered 4\% EDTA for demineralization. Semi-serial histological slices were obtained considering long-axis of the sockets and stained with hematoxylin-eosin and Goldner trichrome. For this, the first slices that presented the entire sockets were selected. Then, every each $30 \mu \mathrm{m}$ slices were obtained to be analyzed. A descriptive histopathological analysis was performed based on the analysis of all slices obtained from each animal of each group, taking into consideration: inflammation, bone formation, maturation and remodeling, bone viability, biofilm adhesion, and overlying mucosa. 


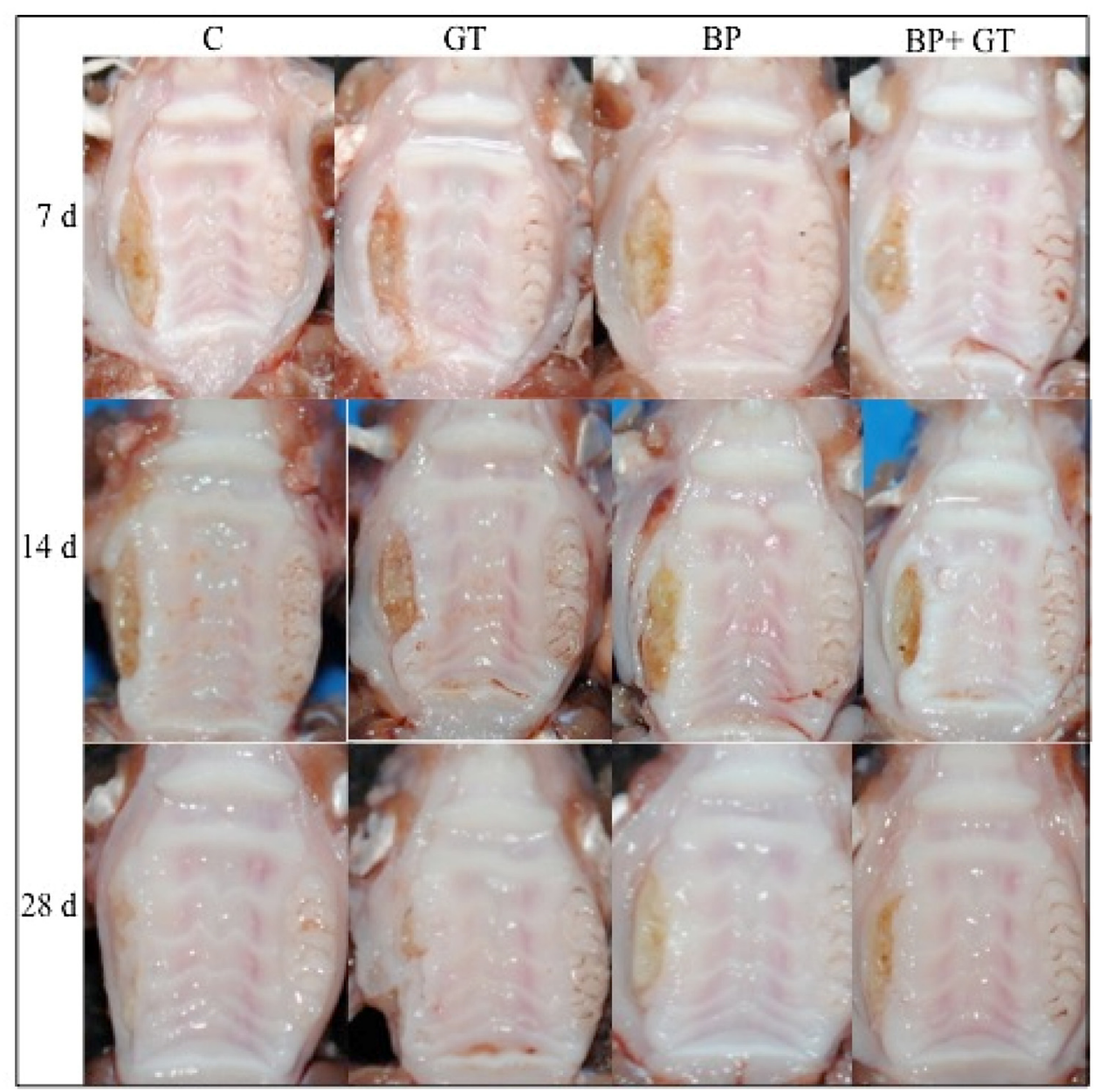

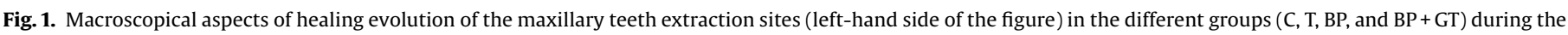
experimental periods ( 7,14 , and 28 days).

\subsection{Immunohistochemistry}

For determination of molecular patterns of bone repair, $3 \mu \mathrm{m}$ sections were depafafinized in xylene and rehydrated in a graded series of ethanol to distilled water, and then immersed in $0.01 \mathrm{M}$ citrate-buffer at $\mathrm{pH} 6.0$ to be heated in a steamer for $30 \mathrm{~min}$. Histological slices were be treated with proteinase $\mathrm{K}$ for $30 \mathrm{~min}$ in room temperature. Endogenous peroxydase was blocked with $2 \%$ peroxyde hydrogen for $10 \mathrm{~min}$ and washed with PBS (phosphate buffer solution). After this period, primary polyclonal antibodies (Santa Cruz Biotechnology, Santa Cruz, CA, EUA) anti-Runx2 (SC8566), anti-RANKL (SC-7627), anti-OPG (SC-21038), anti-OC (SC18319), anti-TRAP (SC-30832) were incubated overnight at $4{ }^{\circ} \mathrm{C}$ and washed for $30 \mathrm{~min}$, three times. The slices were incubated with biotinylated secondary antibody (LSAB, Dakocytomation) for 30 min, washed in PBS and incubated with streptavidin-peroxidase conjugate (LSAB, Dakocytomation) for more $30 \mathrm{~min}$. After, reaction was stained with 3,3'-diaminobenzidine tetrahydrochloride (Sigma Aldrich, St Louis, MO, USA) and counterstained with Harris hematoxylin. For negative control, primary antibody was ommited. Labeling levels for each antibody was determined by semiquantitative analysis, considering the scores from "-" to " +++ " ( $-=$ absent, $+=$ mild, $++=$ moderate, and $+++=$ intense) performed by two expert evaluators in a double-blind system (Bresaola, Matsumoto, Zahoui, Biguetti, \& Nary-Filho, 2016).

\subsection{Morphometric assessment}

A total of six fields of the apical and medium regions of the molar sockets (two for each extracted molar) of each specimen were digitally captured in $40 \times$ magnification (Nikon - Eclipse 80i, Tokyo, Japan) to be analyzed with software Image (National Institutes of Health) (Abramoff, Magalhães, \& Ram, 2004). Acute and chronic inflammation were assessed by the quantification of polymorphonuclear and mononuclear leukocytes in each captured field. The sum of the six fields corresponded to the total number of each type of leukocyte, and the values were statistically treated.

Table 1

Phenolic compounds concentration expressed as gallic acid obtained from Folin Ciocalteu method in different extraction process.

\begin{tabular}{llll}
\hline Temperature $\left({ }^{\circ} \mathrm{C}\right)$ & Time (minutes) & Average & Conc $(\mathrm{mg} / 100 \mathrm{ml})$ \\
\hline 40 & 5 & 0,207 & 116,02 \\
& 10 & 0,211 & 117,19 \\
60 & 5 & 0,299 & 142,27 \\
& 10 & 0,267 & 133,29 \\
100 & 5 & 0,316 & 147,33 \\
& 10 & 0,303 & 143,49 \\
\hline
\end{tabular}




\subsection{Statistical assessment}

Differences among data sets were statistically analyzed by Kruskal-Wallis (followed by the Dunn's test), since data did not fit in the distribution of normality. Values of $p<0.05$ were considered statistically significant. All statistical tests were performed using the GraphPad Prism 5.0 software (GraphPad Software Inc., San Diego, CA, USA).

\section{Results}

\subsection{Total phenol compounds analysis from $1 \%$ green tea infusion}

The higher concentration of phenolic compounds obtained from the three different temperatures and time was when tea was prepared under $100^{\circ} \mathrm{C}$ for $5 \mathrm{~min}$, as shown in Table 1 .

\subsection{Histopathological analysis}

At day 7, alveolar sockets of all groups were exposed, with no soft tissue coverage. Sockets of Control group healed uneventfully, presenting histopathological patterns according to normal chronology (Niccoli-Filho, Okamoto, Cardenuto, \& Picon, 1993). However, at day 7, GT group presented cervical exposed bone with no osteocytes, and intense neutrophilic infiltrate. Minimal osteogenic activity was noted in apical region. Infected sockets were observed in BP and BP + GT groups at the same period, filled by a highly inflammed granulation tissue. Intriguingly, at day $14 \mathrm{GT}$, $\mathrm{BP}$ and $\mathrm{BP}+\mathrm{GT}$ groups were quite similar, with the persistance of non-viable bone tissue and osteoclastic activity and intense mononuclear and neutrophil infiltrate, along with the biofilm formation inside the sockets. At the 28th day, GT group sockets partially covered by the mucosa, and a discrete new bone formation at the apical region. At the same period BP and BP+GT groups still presented exposed bone surfaces, persistance of biofilm adhered to the apical, medium and cervical regions, and severe neutrophilic infiltrate. Histopathological pattern of the groups in the different periods are demonstrated in Fig. 2.

\subsection{Histomorphometry}

At days 7 and 14 no significant differences in the number of polymorphonuclear leukocytes were detected among the groups. At day $28, \mathrm{BP}(784 \pm 219)$ and BP + GT groups $(636 \pm 194)$ presented a significant increased number of neutrophils in comparison to $C$ group $(67 \pm 30)$ (Fig. 3a). In relation to mononuclear leukocytes, a decrease of these cells in GT $(285 \pm 103)$ and BP + GT groups $(418 \pm 27)$ in comparison to the $C$ group $(1033 \pm 566)$ was detected at the first period ( 7 days). No significant differences were detected among the groups in the following periods (Fig. $3 \mathrm{~b}$ ).

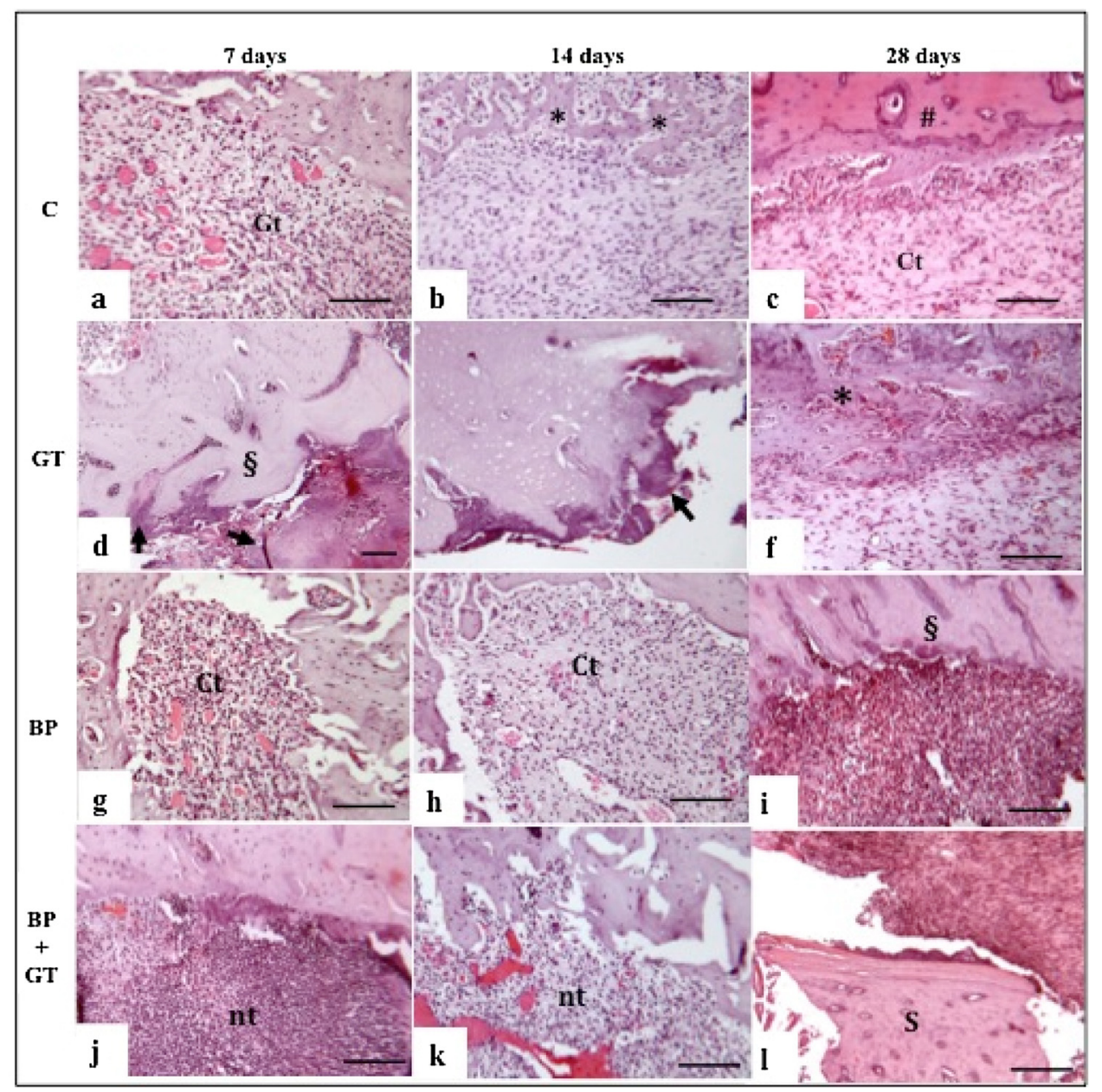

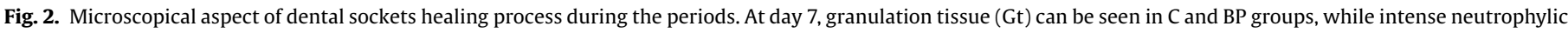

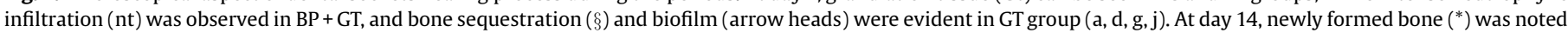

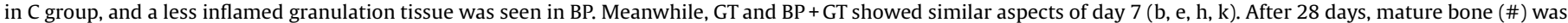

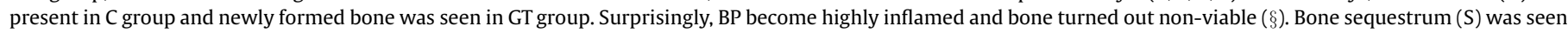
in $\mathrm{BP}+\mathrm{GT}$ group $(\mathrm{H} \& \mathrm{E}$; bar $=200 \mu \mathrm{m})$. 
a

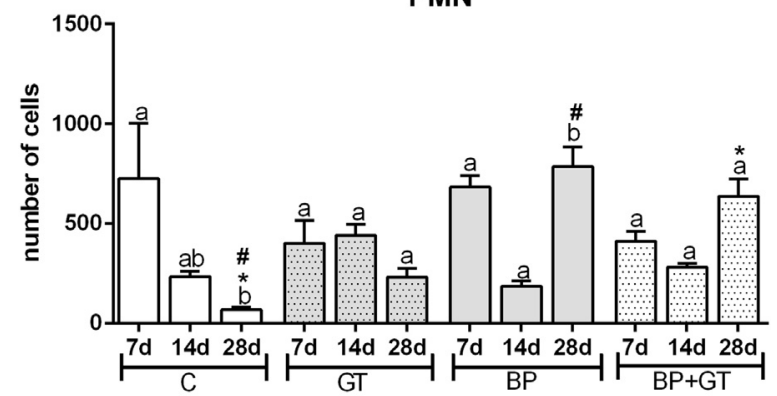

b

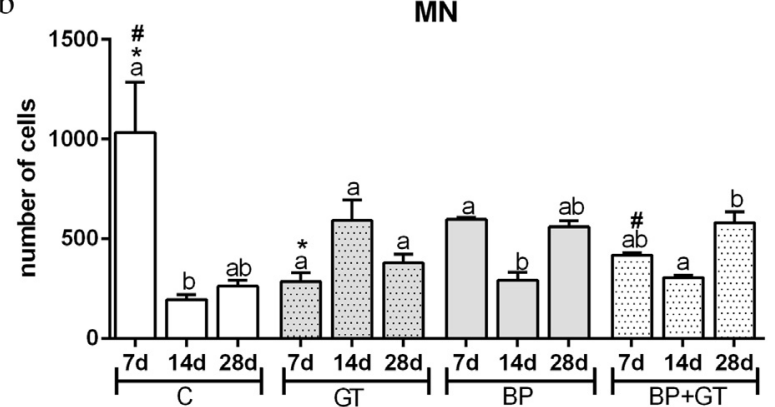

Fig. 3. (a) Haistomorphometric analysis of PMN. Quantity of cells is presented as mean and standard deviation SD. Different letters indicate statistically significant differences $(p<0.05)$ among the periods considering the same group. Symbols indicate statistically significant diferences $(\mathrm{p}<0.05)$ among the groups considering a specific period. (b) Hbistomorphometric analysis of MN. Quantity of cells is presented as mean and standard deviation SD. Different letters indicate statistically significant differences $(\mathrm{p}<0.05)$ among the periods considering the same group. Symbols indicate statistically significant diferences $(\mathrm{p}<0.05)$ among the groups considering a specific period.

\subsection{Immunohistochemistry}

Labeling score for each protein is shown in Table 2, and figures of immunohistochemical labeling at day 28 are represented in Fig. 4. A weak Runx-2 protein immunolabeling, indicative for osteoblast differentiation, was observed only in the osteoblasts of GT group, with no labeling in the other groups. At day 14, group C presented a weak immunolabeling, increasing at day 28 in all groups, except in BP+GT group, which remained weak. Strong initial RANKL labeling was observed when zoledronic acid and green tea was administered, decreasing at day 14 in all groups and increasing again in groups BP and BP+GT. Meanwhile, OPG labeling was generally absent or weak in all groups at days 7 and 14 , becoming moderate in BP group at day 28. All groups presented similar labeling pattern for TRAP at day 7 , when it was moderate, decreasing at the following period. At day 28 , labeling peaks ware noticed in BP and BP+GT groups. However, despite the strong immunostaining in these groups, TRAP positive cells were more concentrated in granulation tissue, and not on bone surface as they were in C and GT groups.

\section{Discussion}

It is clear that green tea consumption did not play a beneficial role during socket healing, and that BP administration did negatively interfere in socket repair in the present study, resulting in a bone condition compatible to osteonecrosis, as revealed by histopathologic analysis. Concentration and via of administration of GT was chosen considering its beneficial systemic effects on hepatic and oxidative DNA damage in a high-cholesterol diet model in rats (de Moraes et al., 2004), along with the results obtained in the present study. It is important to emphasize that the
Table 2

Mean scores assigned for RUNX-2, OPG, RANKL, TRAP, and OC according to the analyzed periods in all groups.

\begin{tabular}{|c|c|c|c|c|c|}
\hline & RUNX-2 & OPG & RANKL & TRAP & OC \\
\hline \multicolumn{6}{|l|}{7 days } \\
\hline $\mathrm{C}$ & - & - & + & ++ & - \\
\hline GT & + & + & +++ & ++ & - \\
\hline $\mathrm{BP}$ & - & - & ++ & ++ & - \\
\hline $\mathrm{BP}+\mathrm{GT}$ & - & + & +++ & ++ & - \\
\hline \multicolumn{6}{|l|}{14 days } \\
\hline $\mathrm{C}$ & + & ++ & + & + & - \\
\hline GT & - & + & + & + & - \\
\hline BP & - & + & + & - & - \\
\hline $\mathrm{BP}+\mathrm{GT}$ & - & + & + & + & - \\
\hline \multicolumn{6}{|l|}{28 days } \\
\hline C & ++ & - & + & ++ & + \\
\hline GT & ++ & + & + & ++ & + \\
\hline $\mathrm{BP}$ & ++ & ++ & ++ & +++ & - \\
\hline $\mathrm{BP}+\mathrm{GT}$ & + & + & ++ & +++ & + \\
\hline
\end{tabular}

difficult in working with this kind of beverage is that a number of conditions can influence final concentration of phenolic compounds after tea preparation, from leaves manufacturing to their particle size, and even the product presentation, inside bags (teabags) or not, since packing material can also influence tea quality (Astill, Birch, Dacombe, Humphrey, \& Martin, 2001).

However, despite the reliable evidences of green tea antinflammatory action in a number of systemic and soft tissue pathologies (Hsu, 2005), and its beneficial effects on bone health in general (Shen et al., 2011), little is known about green tea systemic effects on bone healing, and especially dental socket healing (Mah et al., 2014). In the present study, green tea preparation was chosen to get the closest as possible of general population practice, and also considering data collected from longitudinal studies, which show the benefits of tea drinking on bone density, especially in elderly women (Devine et al., 2007; Hegarty, May, \& Khaw, 2000)]. Our main objective was to observe socket healing process in vivo of rodents under BP therapy and regular ingestion of green tea. The most important observation is that from the obtained results, it is evident that the association of both substances seemed to cause some disturbance in bone healing as shown by inflammatory pattern and immunohistochemistry analysis.

When comparing PMN cells quantity, no significant differences were detected between GT and BP except for day 28 when it was higher in BP group in comparison to $C$ group. However, the analysis of inflammation dynamics during the periods showed that GT group maintained a balanced number of both types of leukocytes, the same observed when it was associated with BP, while an increase was seen when BP was administered alone. From these results, one could conclude that biodisponibility of oral intake of green tea and its antiinflammatory capacity may probably be reduced, but still enough to play a minimal influence on inflammation. It can be confirmed by the analysis of the last experimental period, when $C$ and GT groups ended up presenting no significant differences in the number of both types of leukocytes, although GT socket healing was poorer in comparison to $\mathrm{C}$ ones.

Although it is known that systemic alterations and the use of concomitante drugs may favor the development of the necrosis of the jaws in patients under IV nBPs therapy (Yanık et al., 2006), animal models using healthy rats, male and female, have been proven to induce the disease in different levels, depending on the protocol (Barbra-Recreo, Del Castillo Pardo de Vera, García-Arranz, Yébenes \& Burgueño, 2014). Considering the aim of this study, that was to analyze the effects of the association between GT and BP in teeth extraction model, and not the mandatory induction of 


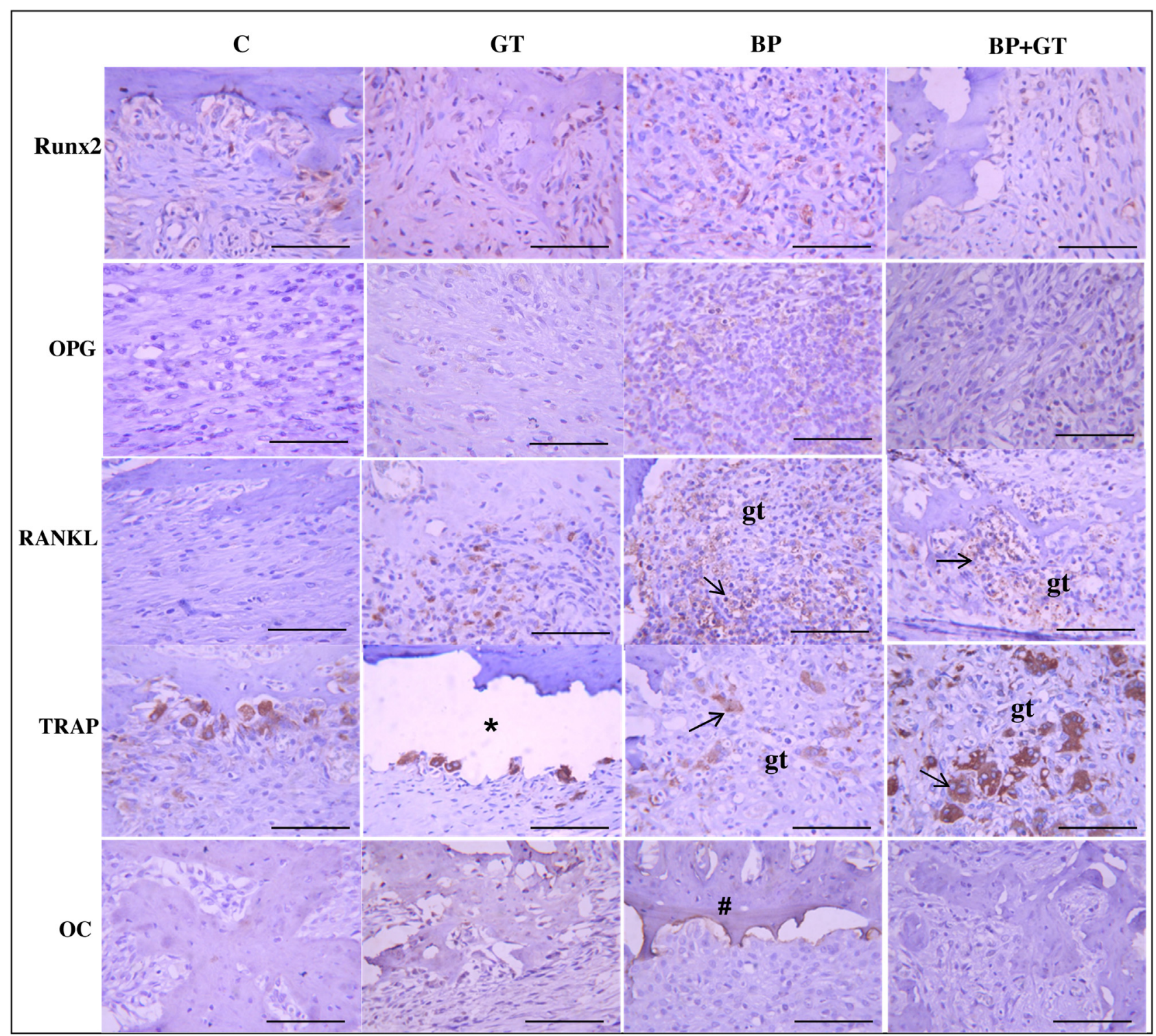

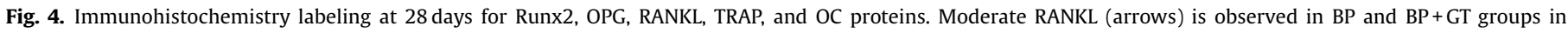

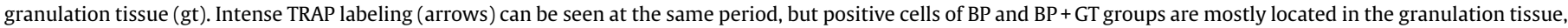
and not on bone surface as in C and GT groups. OC is predominant in old bone (\#) in BP group $\left({ }^{*}=\operatorname{artifact}\right)(\mathrm{bar}=200 \mu \mathrm{m})$.

osteonecrosis for curative purposes, Hokugo et al. (2010) protocol was chosen. Their protocol reaches the closest percentage of osteonecrosis of the jaws (14.3\%) of real clinical practice, which varies from 0.8 to $12 \%$ (Vescovi \& Nammour, 2010).

In our study it was detected intense immunolabeling of TRAP at day 28 in both groups where BP was administered. The evidences of in vitro studies that RANKL and TNF $\alpha$ can avoid the apoptotic effects of etidronate and alendronate on osteoclats (Sutherland, Rogers, Tosh, \& Rogers, 2009) can explain it. Although no similar study on IV nBPs was conducted, but considering that both types of BPs were tested, it can be concluded that this effect can also occur when using zoledronic acid or pamidronate. However, an important observation must be highlighted, which is that in BP and $\mathrm{BP}+\mathrm{GT}$ groups, TRAP positive cells were mostly located in the granulation tissue, and not attached to bone surface. These difuse osteoclasts did not present the active resorption shape, but the intense PMN and MN infiltrate along with histopathological observations and immunolabeling pattern, especially for Runx-2 and $\mathrm{OC}$, suggested active bone resorption and/or inhibition of tissue formation. Although previous study (Lin et al., 2009) had clearly evidenced the action of GT in the inhibition of osteoclastic differentiation via NF- $\kappa$ B in a dose-dependent manner, somehow secretion of RANKL had possibly interfereed in this process. In a recente study (Cardemil, Thomsen, \& Larsson Wexell, 2015), perinecrotic jawbone were analyzed retrieved from patients treated with BPs with and without osteonecrosis of the jaws and healthy patients, revealing no differences in RANKL/OPG ratios among the groups.

\section{Conclusions}

Dental socket healing of rats treated with BP and regular drinking green tea presented no relevant differences in comparison to those treated with BP alone. A slight influence on inflammatory process was noted, but not enough to improve cell activity and healing process.

\section{Conflict of interest}

Authors declare no conflict of interest. 


\section{Acknowledgements}

The authors are grateful to Maira Cristina Rondina Couto for histology and imunohistochemical assistance. This work was funded by Fundação de Amparo à Pesquisa do Estado de São Paulo (FAPESP/SP), grant number 2013/04714-8.

\section{References}

Abramoff, M. D., Magalhaes, P. J., \& Ram, S. J. (2004). Image processing with Image Biophotonics International, 7, 36-42.

Astill, C., Birch, M. R., Dacombe, C., Humphrey, P. G., \& Martin, P. T. (2001). Factors affecting the caffeine and polyphenol contents of black and green tea infusions. Journal of Agricultural and Food Chemistry, 49, 5340-5347.

Barba-Recreo, P., Del Castillo Pardo de Vera, J. L., García-Arranz, M., Yébenes, L., \& Burgueño, M. (2014). Zoledronic acid-related osteonecrosis of the jaws. Experimental model with dental extractions in rats. Journal of Craniomaxillofacial Surgery, 42, 744-750.

Biasotto, M., Chiandussi, S., Zacchigna, S., Moimas, S., Dore, F., Pozzato, G., et al. (2010). A novel animal model to study non-spontaneous bisphosphonates osteonecrosis of jaw. Journal of Oral Pathology and Medicine, 39, 390-396.

Bresaola, M. D., Matsumoto, M. A., Zahoui, A., Biguetti, C. C., \& Nary-Filho, H. (2016) Influence ofrapid- and slow-rate resorption collagen membrane in maxillary sinus augmentation. Clinical Oral Implants Research Epub ahead of print.

Cardemil, C., Thomsen, P., \& Larsson Wexell, C. (2015). Jaw bone samples from bisphosphonate-treated patients: A pilot cohort study. Clinical Implant Dentistry and Related Research, 17(Suppl. 2), e679-e691.

Chen, C. H., Ho, M. L., Chang, J. K., Hung, S. H., \& Wang, G. J. (2005). Green tea catechin enhances osteogenesis in a bone marrow mesenchymal stem cell line. Osteoporosis International, 16, 2039-2045.

Coxon, F. P., \& Rogers, M. J. (2003). The role of prenylated small GTP-binding proteins in the regulation of osteoclast function. Calcified Tissue International, 72, 80-84.

Cremers, S., \& Papapoulos, S. (2011). Pharmacology of bisphosphonates. Bone, 49, 42-49.

de Moraes, B. B., Pasquini, G., Aguiar, O. Jr., Gollücke, A. P., Ihara, S. S., Tenorio, N. M., et al. (2004). Protective effe cts of green tea against hepatic injury induced by high-cholesterol diet in rats: Histopathological analysis, oxidative DNA damage and COX-2 expression. Hepatology International, 5, 965-974.

Devine, A., Hodgson, J. M., Dick, I. M., Prince, R. L., Devine, A., Hodgson, J. M., et al. (2007). Tea drinking is associated with benefits on bone density in older women. American Journal of Clinical Nutrition, 86, 1243-1247.

Fisher, J. E., Rodan, G. A., \& Reszka, A. A. (2000). In vivo effects of bisphosphonates on the osteoclast mevalonate pathway. Endocrinology, 141, 4793-4796.

Gutta, R., \& Louis, P. J. (2007). Bisphosphonates and osteonecrosis of the jaws: Science and rationale. Oral Surgery Oral Medicine Oral Pathology Oral Radiology and Endodontics, 104, 186-193.

Hadji, P. (2011). Managing bone health with zoledronic acid: A review of randomized clinical study results. Climacteric, 14, 321-332.

Hegarty, V. M., May, H. M., \& Khaw, K. T. (2000). Tea drinking and bone mineral density in older women. American Journal of Clinical Nutrition, 71, 1003-1007.

Hokugo, A., Christensen, R., Chung, E. M., Sung, E. C., Felsenfeld, A. L., Sayre, J. W., et al. (2010). Increased prevalence of bisphosphonate-related osteonecrosis of the jaw with vitamin D deficiency in rats. Journal of Bone and Mineral Research, 25 1337-1349.

Hsu, U. (2005). Green tea and the skin. Journal of the American Academy of Dermatology, 52, 1049-1059.

Lawson, M. A., Xia, Z., Barnett, B. L., Triffitt, J. T., Phipps, R. J., Dunford, J. E., et al. (2010). Differences between bisphosphonates in binding affinities for hydroxyapatite. Journal of Biomedical Materials Research: Part B: Applied Biomaterials, 92, 149-155.

Lin, R. W., Chen, C. H., Wang, Y. H., Ho, M. L., Hung, S. H., Chen, I. S., \& Wang, G. J. (2009). (-)-Epigallocatechin gallate inhibition of osteoclastic differentiation via
NF-kappaB. Biochemical and Biophysical Research Communications, 379, 1033 1037.

Loiselle, A. E., Jiang, J. X., \& Donahue, H. J. (2013). Gap junction and hemichannel functions inosteocytes. Bone, 4, 205-212.

Mah, Y. J., Song, J. S., Kim, S. O., Lee, J. H., Jeon, M., Jung, U. W., et al. (2014). The effect of epigallocatechin-3-gallate (EGCG) on human alveolar bone cells both in vitro and in vivo. Archives of Oral Biology, 59, 539-549.

Marino, K. L., Zakhary, I., Abdelsayed, R. A., Carter, J. A., O'Neill, J. C., Khashaba, R. M., et al. (2012). Development of a rat model of bisphosphonate-related osteonecrosis of the jaw (BRONJ). Journal of Oral Implantology, 38, 511-518.

Marx, R. E., Sawatari, Y., Fortin, M., \& Broumand, V. (2005). Bisphosphonate-induced exposed bone (osteonecrosis/osteopetrosis) of the jaws: Risk factors, recognition, prevention, and treatment. Journal of Oral and Maxillofacial Surgery, $63,1567-1575$.

Marx, R. E. (2003). Pamidronate (Aredia) and zoledronate (Zometa) induced avascular necrosis of the jaws: A growing epidemic. Journal of Oral and Maxillofacial Surgery, 61, 1115-1117.

Nakagawa, T., Ohta, K., Kubozono, K., Ishida, Y., Naruse, T., Takechi, M., et al. (2015). Zoledronate inhibits receptor activator of nuclear factor kappa-B ligandinduced osteoclast differentiation via suppression of expression of nuclear factor of activated T-cell c1 and carbonic anhydrase 2. Archives of Oral Biology, 60, 557-565.

Niccoli-Filho, W. D., Okamoto, T., Cardenuto, N., \& Picon, L. C. (1993). The effect of green helium-neon laser on the healing of extraction wounds: Histological study in rats. Revista de Odontologia da UNESP, 22, 213-221.

Oka, Y., Iwai, S., Amano, H., Irie, Y., Yatomi, K., Ryu, K., et al. (2012). Tea polyphenols inhibit rat osteoclast formation and differentiation. Journal of Pharmacological Science, 118, 55-64.

Rogers, M. J., Crockett, J. C., Coxon, F. P., \& Mönkkönen, J. (2011). Biochemical and molecular mechanisms of action of bisphosphonates. Bone, 49, 34-41.

Russell, R. G. (2011). Bisphosphonates: The first 40 years. Bone, 49, 2-19.

Sato, K., \& Takayanagi, H. (2006). Osteoclasts, rheumatoid arthritis, and osteoimmunology. Current Opinion Rheumatology, 18, 419-426.

Senel, F. C., Kadioglu Duman, M., Muci, E., Cankaya, M., Pampu, A. A., Ersoz, S., et al. (2010). Jaw bone changes in rats after treatment with zoledronate and pamidronate. Oral Surgery Oral Medicine Oral Patholology Oral Radiology and Endodontics, 109, 385-391.

Sharma, D., Hamlet, S., Petcu, E., \& Ivanovski, S. (2013). Animal models for bisphosphonate-related osteonecrosis of the jaws-an appraisal. Oral Diseases, 19, 747-754.

Shen, C. L., Yeh, J. K., Cao, J. J., Chyu, M. C., \& Wang, J. S. (2011). Green tea and bone health: Evidence from laboratory studies. Pharmacology Research, 64, 155-161.

Singh, R., Akhtar, N., \& Haqqi, T. M. (2010). Green tea polyphenol epigallocatechin-3gallate: Inflammation and arthritis. Life Sciences, 86, 907-918.

Sutherland, K. A., Rogers, H. L., Tosh, D., \& Rogers, M. J. (2009). RANKL increases the level of Mcl-1 in osteoclasts and reduces bisphosphonate-induced osteoclast apoptosis in vitro. Arthritis Research E Therapy, 11, R58.

Tipoe, G. L., Leung, T. M., Hung, M. W., \& Fung, M. L. (2007). Green tea polyphenols as an anti-oxidant and anti-inflammatory agent for cardiovascular protection. Cardiovascular \& Hematological Disorders-Drug Targets, 7, 135-144.

Tomata, Y., Kakizaki, M., Nakaya, N., Tsuboya, T., Sone, T., Kuriyama, S., et al. (2012). Green tea consumption and the risk of incident functional disability in elderly Japanese: The Ohsaki Cohort 2006 Study. American Journl of Clinical Nutrition, 95, 732-739.

Vescovi, P., \& Nammour, S. (2010). Bisphosphonate-Related Osteonecrosis of the Jaw (BRONJ) therapy. A critical review. Minerva Stomatology, 59, 181-213.

Yanık, S., Aras, M. H., Erkılıc, S., Bozdağ, Z., Demir, T., \& Cetiner, S. (2006). Histopathological features of bisphosphonates related osteonecrosis of the jaw in rats with and without vitamin d supplementation. Archives of Oral Biology, 65, 59-65.

Yun, J. H., Pang, E. K., Kim, C. S., Yoo, Y. J., Cho, K. S., Chai, J. K., et al. (2004). Inhibitory effects of green tea polyphenol (-)-epigallocatechin gallate on the expression of matrix metalloproteinase- 9 and on the formation of osteoclasts. Journal of Periodontal Research, 39, 300-307. 